\title{
Factores de riesgo asociados a patologías musculoesqueléticas en deportistas con pie cavo anterior a través de estudios de baropodometría
}

\author{
Risk factors associated to musculoskeletal pathologies in athletes \\ with cavus foot through baropodometric studies \\ Orozco-Villaseñor SL, * Mayagoitia-Vázquez JJ, ${ }^{\ddagger}$ \\ Miguel-Andrés I, ${ }^{\S}$ De la Cruz-Alvarado KD, ${ }^{ף}$ Villanueva-Salas R\| \\ Centro de Innovación Aplicada en Tecnologías Competitivas (CIATEC, A.C.).
}

RESUMEN. Introducción: El pie cavo es una patología musculoesquelética con un aumento del arco medial del pie. La etiología del pie cavo aún es incomprensible, está relacionada con afecciones neurológicas, enfermedad de Charcot-Marie-Tooth, ataxia de Friedreich y parálisis cerebral. El objetivo de esta investigación fue analizar la distribución de la presión plantar en atletas jóvenes con pie cavo. Material y métodos: Se reclutaron 83 atletas jóvenes de entre nueve y 20 años de edad, que presentaban patología de pie cavo. La masa y el promedio de altura fueron $56.9 \pm 12.36 \mathrm{~kg}$ y $1.61 \pm 0.10 \mathrm{~m}$, respectivamente. La distribución de la presión plantar de los pies se registró durante condiciones estáticas. Se compararon las presiones del antepié y el retropié. Resultados: La distribución de la presión plantar se categorizó en tres grupos. En el primer grupo los participantes presentaron mayor presión en la parte anterior del pie; en el segundo grupo los atletas mostraron una presión similar en la región posterior y anterior de los pies y en el último los sujetos revelaron una mayor presión en el retropié. Para ser considerado en uno de los tres grupos, la diferencia de presión entre la parte posterior y anterior del
ABSTRACT. Introduction: The cavus foot is a musculoskeletal pathology with an increase of the medial arch of the concavity of the foot. The etiology of the cavus foot is still enigmatic, it is related with neurologic conditions, Charcot-Marie-Tooth disease, Friedreich's ataxia, and cerebral palsy. The aim of this research was to analyze the plantar pressure distribution of the feet on young athletes with cavus foot. Material and methods: Eighty-three young athletes between nine and 20 years old, that presented cavus feet pathology were recruited. The mass and height average were $56.9 \pm 12.36 \mathrm{~kg}$ and 1.61 $\pm 0.10 \mathrm{~m}$ respectively. Plantar pressure distribution of the feet was recorded during static conditions. The hindfoot and forefoot pressure were compared in each foot. Results: The plantar pressure distribution were categorized in three groups. In the first group the participants presented higher pressure in the hindfoot than forefoot, in the second group, the athletes showed similar pressure in the posterior and anterior region of the feet and in the last one, the subjects revealed higher pressure in the forefoot. To be considered in one of the three groups, the difference of pressure between

Nivel de evidencia: III

* Asesor de Biomecánica, Cirujano de Pie y Tobillo, CIATEC, A.C. México.

* Coordinador del Laboratorio de Biomecatrónica, CIATEC, A.C. México.

$\S$ Doctor Investigador en Biomecánica, CIATEC, A.C. México.

"Ingeniería Biomédica, CIATEC, A.C. México.

" Médico Residente de $4^{\circ}$ año de Ortopedia del Hospital General de León. México.

Correspondencia:

Dr. Sergio Luis Orozco-Villaseñor

Omega No. 201, Col. Industrial Delta, CP 37545, León, Guanajuato, México.

E-mail: sorozco@ciatec.mx

Recibido: 06-05-2019. Aceptado: 04-06-2021.

Citar como: Orozco-Villaseñor SL, Mayagoitia-Vázquez JJ, Miguel-Andrés I, De la Cruz-Alvarado KD, Villanueva-Salas R. Factores de riesgo asociados a patologías musculoesqueléticas en deportistas con pie cavo anterior a través de estudios de baropodometría. Acta Ortop Mex. 2021; 35(4): 317-321. https://dx.doi.org/10.35366/103310

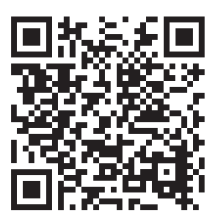


pie se estableció en 16\%. Conclusión: Muchos trastornos musculoesqueléticos en el cuerpo humano son de origen biomecánico y están relacionados con la anatomía del pie. El pie cavo es una patología con alta prevalencia en atletas y está relacionada con las fuerzas mecánicas sobre los pies en condiciones dinámicas.

Palabras clave: Biomecánica, presión plantar, fuerza mecánica, pie cavo.

\section{Introducción}

El pie normal es definido como aquel cuyo ángulo de Moreau-Costa-Bartani tiene valores de 120-130 y el ángulo de Kite de $15-20^{\circ}$. Por otro lado, los arcos longitudinales del pie van decreciendo progresivamente de 18 a $25^{\circ}$ para el primer radio y hasta $5^{\circ}$ para el quinto. Pequeñas alteraciones en cualquiera de estos grados, incluso en ausencia de sintomatología, no siempre son consideradas patológicas. ${ }^{1}$ Las patologías del pie son de origen biomecánico y se asocian con el tipo de pie, éste es un concepto clínico cuyo objetivo es simplificar la complejidad anatómica del pie humano. ${ }^{2}$

Al aumento anormal de la cavidad plantar se le denomina pie cavo, en el cual existe una aproximación de las áreas anteriores y posteriores, siendo una anormalidad en el arco longitudinal medio. Las deformidades se pueden clasificar según su gravedad de desalineación que va desde el pie cavo sutil y flexible, hasta una deformidad fina y severa. ${ }^{3}$ Dependiendo del sitio de la deformidad, según su posición en el plano sagital, se tienen tres tipos de pie cavo: anterior, posterior o mixto. ${ }^{3,4}$

Esta patología se asocia con ciertas enfermedades neuropatológicas debido al desequilibrio muscular paralítico o espático, algunas de ellas son las neuropatías hereditarias sensitivomotoras como la enfermedad de Charcot-Marie-Tooth y atrofias musculares espinales distales. También existe pie cavo idiopático, hipertónico (en deportistas y bailarinas), congénito y secundario a traumatismo.

Las causas traumáticas son poco comunes, sin embargo, se pueden encontrar fracturas tratadas inapropiadamente, subluxación de huesos del tarso o cicatrices por quemaduras en la planta del pie. Una de las causas es el desequilibrio de los músculos antagonistas: peroneo largo y tibial anterior. Los pacientes con pie cavo a menudo presentan pantorrillas estrechas, gastrocnemio corto y estrecho que conduce el aumento de presiones plantares en antepié y fascia plantar, actuando como fuerzas inversoras de deformación del retropié. ${ }^{3}$

Se estima que la prevalencia de pie cavo incrementa con la edad, por lo que existe mayor prevalencia en una edad adulta. En un estudio desarrollado por Espinoza y colaboradores se encontró una prevalencia de pie cavo de $11.6 \%$ en niños y $14.4 \%$ en niñas en una edad entre seis y 12 años. the posterior and anterior part of the foot was established at $16 \%$. Conclusion: Many musculoskeletal disorders in the human body are biomechanical in origin and related with foot anatomy. The cavus foot is a pathology with high prevalence in athletes and it is related with the mechanical forces over the feet during dynamic conditions.

Keywords: Biomechanics, plantar pressure, mechanical strength, cavus foot.
Mientras que en otros estudios en niños de la misma edad se han encontrado porcentajes de prevalencia de $35 \%$. 4,5,6,7,8,9 No obstante, otros autores han reportado porcentajes de prevalencia por debajo de $2 \%$ en niños de entre cinco y seis años de edad. ${ }^{10}$ Uno de los dolores asociados a esta patología es el dolor lumbar (DL), siendo la primera causa de discapacidad y uno de los principales contribuyentes a la carga de la enfermedad. El DL crónico está asociado a altos niveles de dolor, discapacidad, depresión y calidad de vida reducida; por lo que el empleo de órtesis en los pies es uno de los muchos tratamientos que reduce la pronación excesiva o anormal del pie, logrando la alteración cinemática de las estructuras proximales. ${ }^{11}$

Por otro lado, las radiografías son esenciales en una planificación quirúrgica para poder identificar la deformidad y cuantificar el grado de corrección. Se incluyen tres vistas: la vista lateral de tobillo y pie que soportan el peso y permiten medir el pie cavo, la vista frontal muestra la deformación del retropié y la vista dorsoplantar del antepié muestra la aducción del antepié y la apertura de la placa metatarsiana. Las medidas geométricas que permiten la cuantificación de la deformación del pie cavo son: el ángulo del arco medial (Djian-Annonier) menor a $120^{\circ} \mathrm{y}$ el ángulo de Hibbs siendo de más de $45^{\circ}$. $^{3}$

Una vez conociendo los efectos colaterales del pie cavo, el objetivo del presente trabajo es llevar a cabo el análisis de la distribución de la presión plantar en jóvenes atletas con la patología del pie cavo anterior.

\section{Material y métodos}

Se realizó un estudio baropodométrico en el laboratorio de biomecatrónica del CIATEC, A.C., en el período comprendido de 2017-2018. Se obtuvo una muestra de 83 pacientes con alteraciones musculoesqueléticas de tipo pie cavo anterior.

Todos los participantes fueron informados sobre el protocolo y procedimiento experimental. Se obtuvo una historia clínica ortopédica completa, una exploración física para determinar factores asociados en los atletas, así como conocer lesiones previas asociadas al deporte.

Dentro de los criterios de inclusión se consideraron todos los pacientes mexicanos por nacimiento, atletas de alto rendimiento de diversas disciplinas, edad comprendida entre 
los nueve y 20 años de edad, sin antecedentes quirúrgicos previos en ambos pies.

Se llevó a cabo un análisis estadístico de los resultados obtenidos por las pruebas biomecánicas en todos los atletas. Donde se obtuvo un promedio de masa y altura de $56.9 \pm$ $12.36 \mathrm{~kg}$ y $1.61 \pm 0.10 \mathrm{~m}$, respectivamente.

La distribución de la presión plantar de los pies se registró durante condiciones estáticas, se coloca a los participantes en la plataforma de presión (Sensormedica, Guidonia Montecelio, Roma, Italia) en posición de pie, posteriormente se realiza el registro durante 10 segundos. Se analizó la distribución de la presión plantar de los pies y se compara la presión del antepié y retropié. Los datos fueron analizados mediante estadística descriptiva.

\section{Resultados}

Se estudiaron 83 pacientes con alteraciones musculoesqueléticas de tipo pie cavo anterior, de los cuales se incluyen 60 (72.29\%) pacientes del sexo femenino y $23(27.71 \%)$ del sexo masculino. Con edades comprendidas entre nueve y 20 años.

Los resultados obtenidos de la distribución de la presión plantar fue clasificada en tres grupos. En el primer grupo se encuentran aquellos pacientes que presentan una mayor presión en la zona del antepié; en el segundo grupo, se encuentran los pacientes que mostraron una presión equilibrada entre la zona del retropié y antepié $(<16 \%)$, es importante mencionar que la distribución de la carga en un pie normal del antepié es de $45 \%$, mientras que del retropié es de $55 \%$ con una tolerancia de $\pm 3 \%$; en cuanto al tercer grupo se encuentran los pacientes que presentan mayor presión en el retropié. Para considerar a una persona dentro de estos grupos, la diferencia entre el retropié y antepié fue establecida en $16 \%$, como se muestra en la Figura 1.

Se realizó el análisis tanto para el pie derecho como para el izquierdo, en cuanto al pie derecho $60.2 \%$ de los pacientes presentan una mayor presión en zona del retropié, $33.7 \%$ se encuentran en el rango que es considerado normal y $6 \%$ mostró una mayor presión en la zona de antepié (Figura 2).

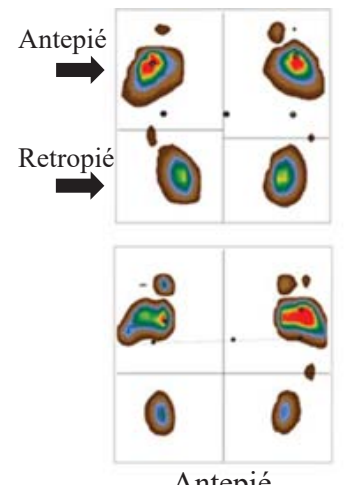

Antepié

Diferencia $>16 \%$

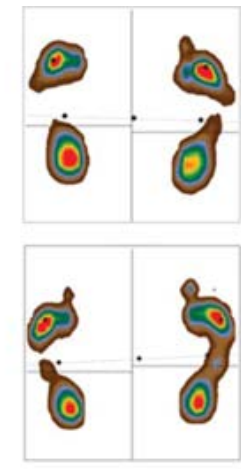

Normal

Diferencia $<16 \%$

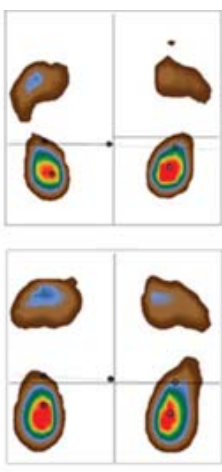

Retropié Diferencia $>16 \%$
Figura 1: Distribución de la presión plantar. Exceso de presión en antepié, normal y retropié.
Distribución de la carga del pie derecho

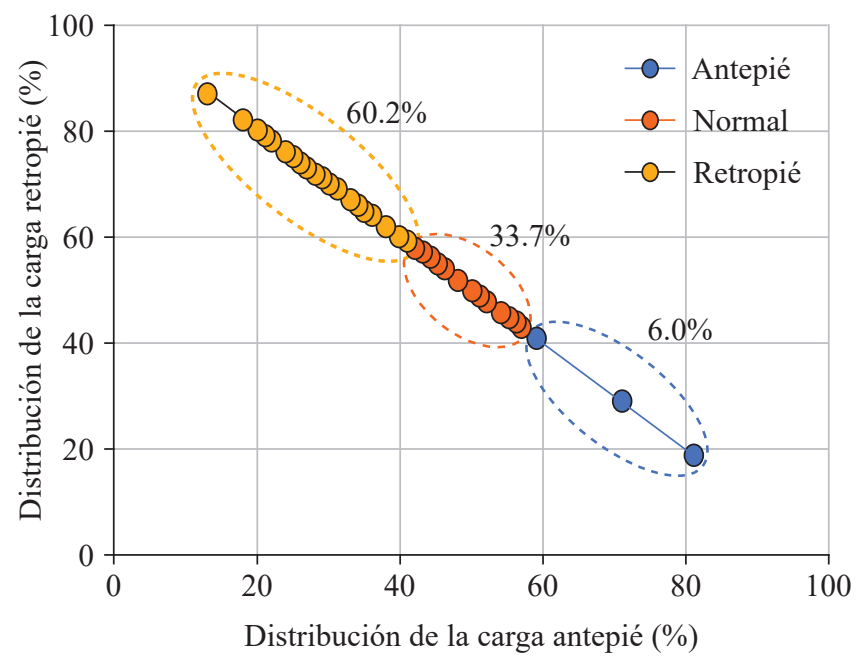

Figura 2: Correlación de la distribución del porcentaje de carga entre retropié y antepié del pie derecho.

Distribución de la carga del pie izquierdo

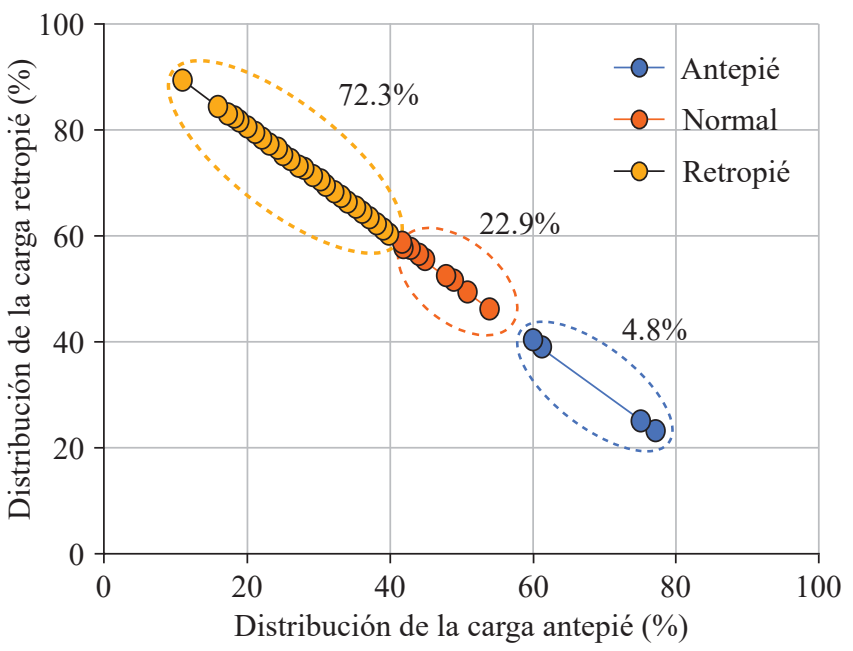

Figura 3: Correlación de la distribución del porcentaje de carga entre retropié y antepié del pie izquierdo.

Por otro lado, en cuanto al pie izquierdo, los resultados obtenidos fueron los siguientes: $72.3 \%$ en retropié, $22.9 \%$ normal y $4.8 \%$ en antepié, como se muestra en la Figura 3. Posteriormente, se realizó el cálculo del promedio del porcentaje de carga del pie derecho, como se muestra en la Figura 4, se obtuvo en el primer grupo un promedio de $68.2 \pm 9.3 \%$ en el antepié y $31.8 \pm 9.3 \%$ en el retropié, en el segundo grupo se encuentra un promedio de $46.7 \pm 4.8 \%$ en el antepié y $53.3 \pm 4.8 \%$ en el retropié, mientras que en el tercer grupo se obtuvo $29.5 \pm 6.8 \%$ en el antepié y $70.5 \pm$ $6.8 \%$ en el retropié. De la misma manera, en la Figura 5 se observa el cálculo del promedio del porcentaje de carga del pie izquierdo, obteniendo en el primer grupo $68.3 \pm 9.0 \%$ en el antepié y $31.8 \pm 9.0 \%$ en el retropié, en el segundo grupo $45.9 \pm 3.6 \%$ en antepié y $54.1 \pm 3.5 \%$ en retropié, mientras 
que en el tercer grupo se obtuvo $28.9 \pm 6.9 \%$ en antepié y $71.1 \pm 6.9 \%$ en retropié.

En relación con el porcentaje de carga, en las gráficas de las Figuras 6 y 7 se observa la superficie de carga del pie derecho e izquierdo, respectivamente. En cuanto a la superficie de carga, tanto en el pie derecho como en el izquierdo, en el mayor número de la población la superficie de carga se encuentra en el retropié, mientras que una cantidad menor posee una superficie de carga en el antepié.

\section{Discusión y conclusión}

La mayoría de los trastornos musculoesqueléticos en el cuerpo humano son de origen biomecánico y se encuentran

Distribución de la carga del pie derecho

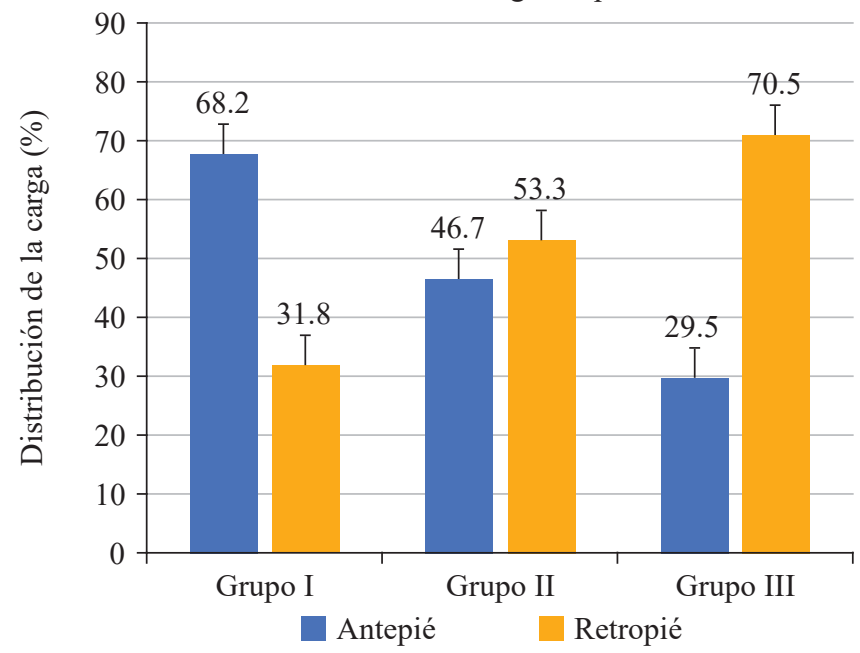

Figura 4: Promedio de la distribución del porcentaje de carga en retropié y antepié del pie derecho. Grupo I: mayor carga en antepié; grupo II: carga equilibrada; grupo III: mayor carga en retropié.

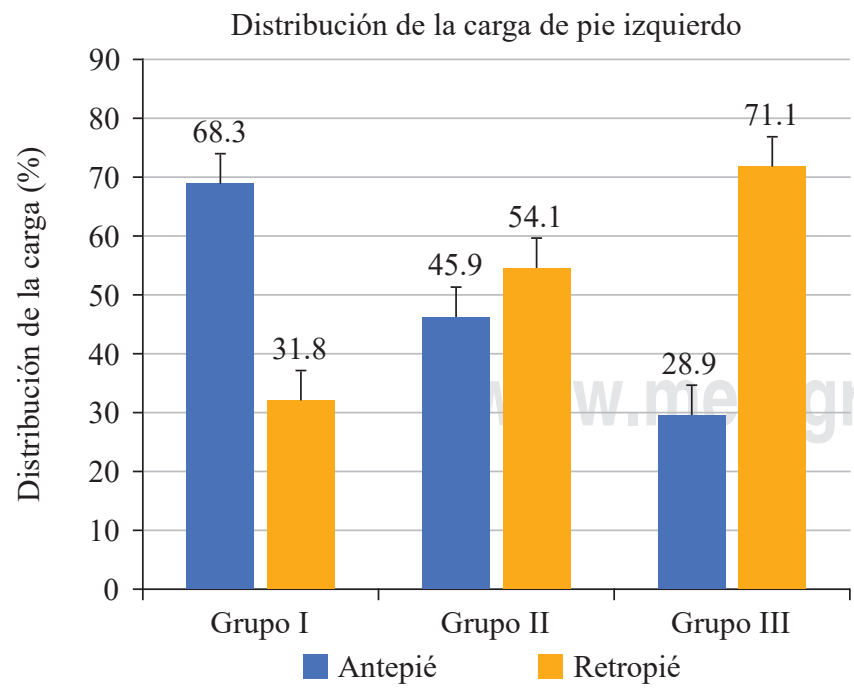

Figura 5: Promedio de la distribución del porcentaje de carga en retropié y antepié del pie izquierdo. Grupo I: mayor carga en antepié; grupo II: carga equilibrada; grupo III: mayor carga en retropié.

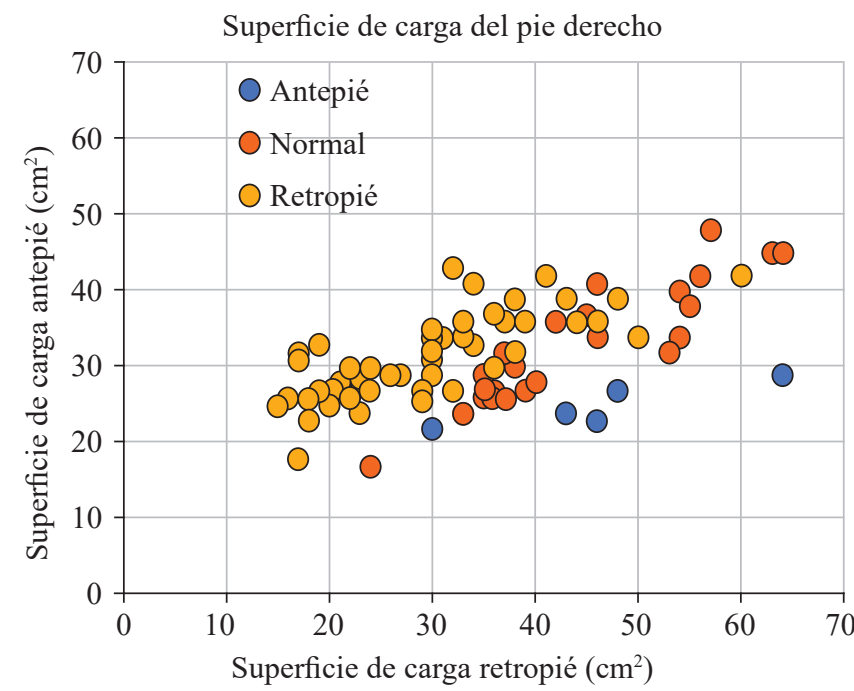

Figura 6: Correlación de la superficie de carga entre el retropié y antepié del pie derecho.

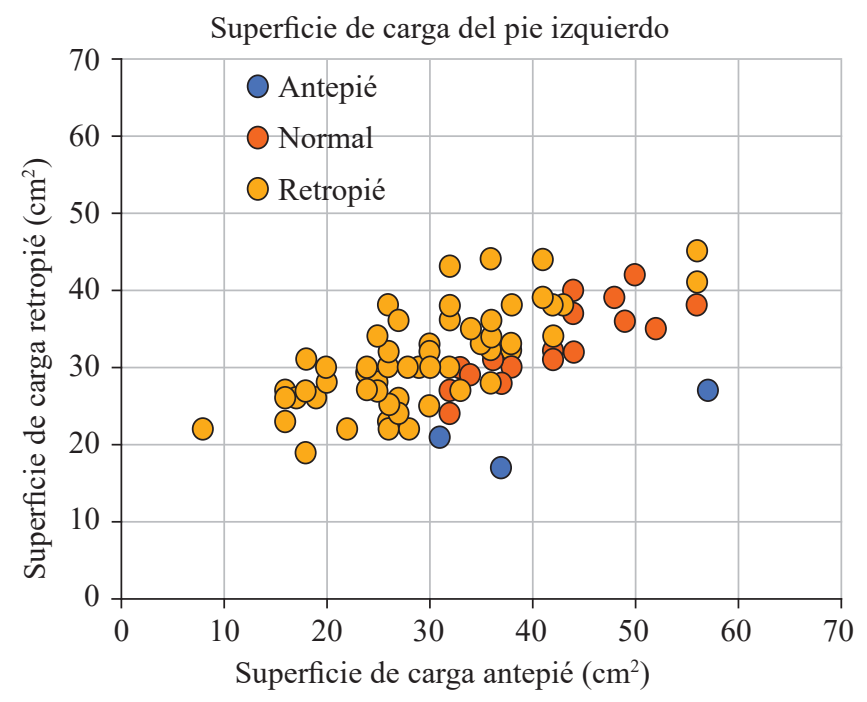

Figura 7: Correlación de la superficie de carga entre el retropié y antepié del pie izquierdo.

relacionados con la anatomía del pie. El análisis de la distribución plantar en atletas nos da información acerca de que el pie cavo es una patología de alta prevalencia en atletas y se encuentran relacionadas con fuerzas mecánicas sobre los pies en condiciones dinámicas. ${ }^{12}$ Esta patología representa un problema de salud pública de suma importancia, ya que afecta de manera negativa en la calidad de vida de las personas que padecen la alteración. ${ }^{13}$

Se encontraron diferencias estadísticamente significativas, se observa que la tendencia del pie cavo es ejercer el mayor porcentaje de la distribución plantar en la zona del retropié (tanto en el pie derecho como en el izquierdo), donde el punto de apoyo se encuentra en la parte posterior en la articulación subastragalina, constituida por el astrágalo y el calcáneo. Los resultados del presente trabajo concuerdan con la idea de que las presiones plantares son 
la señal de posibles alteraciones en el pie que deben ser atendidas de manera inmediata para evitar problemas severos posteriores. ${ }^{14}$

La alta presión en el retropié y antepié podría producir úlceras y/o hiperqueratosis plantar. Además, el pie cavo está asociado con otras lesiones musculoesqueléticas como lo son: esguinces, tendinopatías, fascitis, contracturas musculares y lesiones cartilaginosas; esto significa que mientras más severo sea el pie cavo, más comorbilidades se encontrarán.

\section{Agradecimientos}

Los autores agradecen al Centro de Innovación Aplicada en Tecnologías Competitivas (CIATEC, A.C.) y a la Secretaría de Innovación, Ciencia y Educación Superior (SICES), por el apoyo brindado para la realización y ejecución de este proyecto de investigación.

Referencias

1. Padró ML, Moliné SM. Alteraciones de la bóveda plantar. Rev Española Reumatol. 2003; 30(9): 489-98. Disponible en: https://www. elsevier.es/es-revista-revista-espanola-reumatologia-29-articuloalteraciones-boveda-plantar-13055069

2. Hillstrom HJ, Song J, Kraszewski AP, Hafer JF, Mootanah R, Dufour $\mathrm{AB}$, et al. Foot type biomechanics part 1: structure and function of the asymptomatic foot. Gait Posture. 2013; 37(3): 445-51. doi: 10.1016/j. gaitpost.2012.09.007.

3. Maynou C, Szymanski C, Thiounn A. The adult cavus foot. EFORT Open Rev. 2017; 2(5): 221-9. doi: 10.1302/2058-5241.2.160077.

4. Ledoux WR, Rohr ES, Ching RP, Sangeorzan BJ. Effect of foot shape on the three-dimensional position of foot bones. J Orthop Res. 2006; 24(12): 2176-86. doi: 10.1002/jor.20262.
5. Espinoza-Navarro O, Olivares Urquieta M, Palacios Navarrete P, Robles Flores N. Prevalencia de anomalías de pie en niños de enseñanza básica de entre 6 a 12 años, de colegios de la ciudad de Arica-Chile. Int J Morphol. 2013; 31(1): 162-8. doi: 10.4067/s071795022013000100027.

6. Cala Pérez L, Losa Iglesias ME. Prevalencia de alteraciones musculoesqueléticas en el pie infantil: estudio preliminar. Rev Int Ciencias Podol. 2015; 9(1): 1-16. doi: 10.5209/rev_RICP.2015. v9.n1.47312.

7. Nikolaidou ME, Boudolos KD. A footprint-based approach for the rational classification of foot types in young schoolchildren. Foot. 2006; 16(2): 82-90. doi: 10.1016/j.foot.2006.02.001.

8. Onodera AN, Sacco IC, Morioka EH, Souza PS, de Sá MR, Amadio AC. What is the best method for child longitudinal plantar arch assessment and when does arch maturation occur? Foot (Edinb). 2008; 18(3): 142-9. doi: 10.1016/j.foot.2008.03.003.

9. Rao UB, Joseph B. The influence of footwear on the prevalence of flat foot. A survey of 2300 children. J Bone Joint Surg Br. 1992; 74(4): 525-7. doi: 10.1302/0301-620X.74B4.1624509.

10. Rivera-Saldívar G, Torres-González R, Franco-Valencia M, RíosMonroy R, Martínez-Ramírez F, Pérez-Hernández E, et al. Factores de riesgo asociados a la conformación del arco longitudinal medial y del pie plano sintomático en una población escolar metropolitana en México. Acta Ortop Mex. 2012; 26(2): 85-90.

11. Sadler S, Spink M, Cassidy S, Chuter V. Prefabricated foot orthoses compared to a placebo intervention for the treatment of chronic nonspecific low back pain: a study protocol for a randomised controlled trial. J Foot Ankle Res. 2018; 11: 56. doi: 10.1186/s13047-018-0299-5.

12. Gómez Salazar L, Franco Alvarez JM, Nathy Portilla JJ, Valencia Esguerra EA, Vargas Bonilla DV, Jiménez Hernández L. Características de la huella plantar en deportistas colombianos. Entramado. 2010; 6(2): 158-67.

13. Troiano G, Nante N, Citarelli GL. Pes planus and pes cavus in Southern Italy: a 5 years study. Ann Ist Super Sanita. 2017; 53(2): 142-5. doi: 10.4415/ANN_17_02_10.

14. Deepashini H, Omar B, Paungmali A, Amaramalar N, Ohnmar H, Leonard J. An insight into the plantar pressure distribution of the foot in clinical practice: narrative review. Pol Ann Med. 2014; 21(1): 51-6. doi: 10.1016/j.poamed.2014.03.003. 